Reprod. Nutr. Dévelop., 1988, 28 Suppl. $\mathrm{n}^{\circ} 1,109-110$

\title{
Prévision de la dégradabilité in sacco de l'azote des aliments à partir de leur solubilité dans une solution tampon et de leur dégradation enzymatique
}

Jocelyne AUFRĖRE, Brigitte MICHALET-DOREAU, Dominique CARTAILLER

Unité de la Valeur Alimentaire.

I.N.R.A., Theix, 63122 Ceyrat, France.

Summary. Nitrogen degradability measured by the nylon bag technique was related to solubility and enzymatic degradation. Both methods were good predictors for individual feedstuffs, but enzymatic degradability was superior to solubility for compound feeds.

La méthode in sacco est utilisée pour déterminer la dégradabilité théorique (DT) de l'azote des aliments dans le rumen, et donc pour prévoir la quantité de protéines d'origine alimentaire arrivant au duodénum (Vérité et al., 1987). Des méthodes de laboratoire plus faciles à mettre en œuvre et moins coûteuses peuvent être utilisées pour apprécier des différences de dégradabilité intramatières premières, ou pour estimer la dégradabilité des aliments composés. Le travail présenté a pour but de montrer l'intérêt de deux méthodes : solubilité et hydrolyse enzymatique.

Matériel et méthodes. L'étude a porté :

- sur des matières premières 11 tourteaux de soja, 8 luzernes déshydratées, 15 tourteaux de colza ( 4 ayant subi un traitement technologique), 10 graines de protéagineux (dont 2 extrudées) ;

- sur 20 aliments composés à base de céréales, de tourteaux ou de pulpes, incoporés en proportions variables.

Aussi bien pour les matières premières que pour les aliments composés, la DT présentait une plage de variation importante (tabl. 1).

Pour chaque aliment on a mesuré l'azote total (méthode Kjeldahl), la solubilité de l'azote dans un tampon salive artificielle à $\mathrm{pH} 7$ selon la méthode proposée par Durand (Vérité et Demarquilly, 1978), la dégradabilité in sacco (Michalet-Doreau et al., 1987) et la dégradation enzymatique aux temps $1 \mathrm{~h}$ et $24 \mathrm{~h}$ par une protéase bactérienne extraite de Streptomyces griseus (Sigma type XIV) dans une solution tampon borate-phosphate à $\mathrm{pH} 8$ (Aufrère et Cartailler, in Sauvant et al., 1987).

\section{Résultats et discussion.}

La prévision de la DT pour chacun des aliments simples à partir de la teneur en $\mathrm{N}$ soluble (DT $=\mathrm{a}(\mathrm{N}$ soluble $)+\mathrm{b}$ ) ou à partir de la dégradation enzymatique $(D T=a(D$ enz. $T 1 h)+b(D$ enz. $T 24 h)+c)$ est comparable sauf pour les tourteaux de colza où l'écart-type de la prévision est plus élevé avec la méthode

Reproduction, Nutrition, Développement, 28 , Suppl. $n^{\circ} 1 / 88 \quad 8$ 
TABL. 1. - Liaison entre la dégradabilité théorique et la solubilité ou la dégradation enzymatique de lazote pour les matières premières et les aliments composés.

\begin{tabular}{|c|c|c|c|c|}
\hline \multirow{2}{*}{ Aliments } & \multirow{2}{*}{$\begin{array}{c}\text { Nombre } \\
\text { d'échantillons }\end{array}$} & \multirow{2}{*}{$\begin{array}{l}\text { Dégradabilité } \\
\text { théorique }\end{array}$} & \multirow{2}{*}{$\begin{array}{c}\text { Solubilité } \\
\text { SR }\end{array}$} & \multirow{2}{*}{$\begin{array}{c}\begin{array}{c}\text { Dégradation } \\
\text { enzymatique }\end{array} \\
\text { SR }\end{array}$} \\
\hline & & & & \\
\hline Luzernes déshydratées & 8 & $59,2<\mathrm{DT}<66,1$ & 1,9 & 1,9 \\
\hline Tourteaux de soja & 11 & $61,5<\mathrm{DT}<70,8$ & 1,9 & 2,2 \\
\hline Protéagineux & 10 & $69.7<\mathrm{DT}<93.9$ & 3,7 & 3,9 \\
\hline Tourteaux de colza & 15 & $31,5<\mathrm{DT}<82,9$ & 5,4 & 3,6 \\
\hline Aliments composés: sans $\triangle$ & 20 & $52,9<\mathrm{DT}<82,4$ & 6,5 & 5,0 \\
\hline avec $\wedge$ & & & 6,0 & 4,0 \\
\hline
\end{tabular}

de solubilité (tabl. 1). La précision de la prévision est bonne ce qui est en accord avec les observations de Crawford et al. (1978), Krishnamoorthy (1982), Poos-Floyd et al. (1985). La prévision de la DT pour l'ensemble des aliments composés est plus précise avec la dégradation enzymatique qu'avec la solubilité (tabl. 1). Cependant les aliments contenant plus de $50 \%$ de pulpes de betteraves se comportent différemment des autres aliments composés. Si on en tient compte en introduisant un $\Delta$ dans les équations de prévision (par exemple DT $=$ a ( $N$ soluble) $+b+\Delta$ ) la prévision est améliorée, mais elle l'est plus avec la dégradabilité enzymatique $(S R=4,0)$ qu'avec la solubilité $(S R=6,0)$.

Ces résultats montrent que les deux méthodes de laboratoire étudiées sont comparables pour prévoir les variations de DT intra-matières premières. Cependant, avec la méthode enzymatique, il est possible d'utiliser une équation unique pour prévoir la DT, les différences entre matières premières n'étant significatives que sur l'ordonnée à l'origine, alors qu'avec la méthode de solubilité, ces matières premières ne peuvent être regroupées puisque l'équation de prévision diffère à la fois sur l'ordonnée à l'origine et sur la pente. Cela explique que la prévision de la DT des aliments composés soit meilleure à partir de la méthode enzymatique.

Crawford R. H., Hoover W. H., Sniffen C. J., Crooker B. A., 1978. J. anim. Sci., 46, 1768-1775. Krishnamoorthy U., 1982. Thèse Doctorat, Cornell University.

Michalet-Doreau B., Vérité R., Chapoutot P., 1987. Bull. Tech. CRZV Theix, INRA, 69, 5-7.

Poos-Floyd M., Klopfenstein T., Britton R. A., 1985. J. Dairy Sci., 68, 829-839.

Sauvant D., Aufrère J., Michalet-Doreau B., Giger S., Chapoutot P., 1987. Bull. Tech. CRZV Theix, INRA, 70, 75-89.

Vérité R., Demarquilly C., 1978. In La Vache Laitière, pp. 143-157. Ed. INRA Publ., 78000 Versailles. Vérité R., Michalet-Doreau B., Chapoutot P., Peyraud J. L., Poncet C., 1987. Bull. Tech. CRZV Theix, INRA, 70, 19-34. 